\title{
Successful low-energy cardioversion using a novel biodegradable gel pad: Feasibility of treating postoperative atrial fibrillation in animals
}

Kenji lino, MD, ${ }^{a}$ Nobuhiko Yui, PhD, ${ }^{\text {b }}$ Tooru Ooya, PhD, ${ }^{\text {b }}$ Ryouji Kawabata, ${ }^{\text {b }}$ Shigeyuki Tomita, MD, ${ }^{a}$ and Go Watanabe, MDa

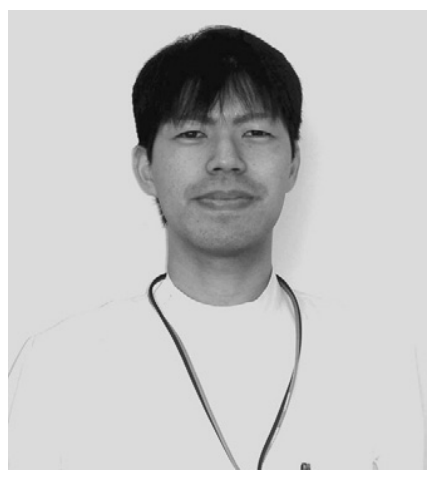

Dr Iino
From the Department of General and Cardiothoracic Surgery, Kanazawa University School of Medicine, ${ }^{\text {a }}$ Kanazawa, Japan; and The School of Materials Science and the 21st Century COE Program, Japan Advanced Institute of Science and Technolo-

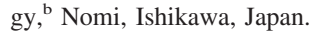

This study was supported by Chisso Co Ltd, Tokyo, Japan.

Received for publication Oct 27, 2006; revisions received Feb 25, 2007; accepted for publication April 26, 2007.

Address for reprints: Kenji Iino, MD, Department of General and Cardiothoracic Surgery, Kanazawa University of Medicine, 13-1 Takaramachi, Kanazawa 920-8641, Japan (E-mail: k-iino@m8.dion.ne.jp).

J Thorac Cardiovasc Surg 2007;134:1519-25 $0022-5223 / \$ 32.00$

Copyright (C) 2007 by The American Association for Thoracic Surgery

doi:10.1016/j.jtcvs.2007.04.073
Objective: Postoperative atrial fibrillation is one of the most frequent complications of cardiac surgery. We developed a novel biodegradable gel pad consisting of biopolymers that directly attach to the myocardium by electrostatic interaction. The present study examines the feasibility and effectiveness of low-energy internal cardioversion using these pads.

Methods: The hearts of 6 pigs were exposed through a median sternotomy under general anesthesia, and 2 monopolar pacing wires were placed on the left pulmonary veins (chest open group). Two biodegradable cardioversion gel pads were placed on the right appendage and the left atria without suturing. All wires were extruded through the skin and secured with a suture. Sustained atrial fibrillation was induced by burst-pacing from the pulmonary veins in continuous $20-\mathrm{ms}$ cycles. Shock intensity started at $0.5 \mathrm{~J}$, and the energy level was increased in 0.5-J increments until cardioversion occurred. This protocol was repeated 5 times per pig. In a second group of 6 pigs (chest closed group), the epicardial cardioversion electrode gel pads and pacing wire electrodes were positioned as described above. Shock intensity was started at $0.5 \mathrm{~J}$. If the shock was unsuccessful, the energy level was increased in 0.5-J increments until 2 consecutive cardioversions were achieved at a single energy level. At postoperative days 1, 3, 5, and 7, the defibrillation threshold was determined with the chest closed. At postoperative day 10 , the cardioversion wires were removed. At predetermined time intervals, the heart was reexposed and the extent of degradation in vivo was visually evaluated and histologically assessed after sacrifice.

Results: All pigs with induced atrial fibrillation were cardioverted to sinus rhythm on the determined postoperative day. The mean energy and lead impedance in the chest open group were $0.65 \pm 0.23 \mathrm{~J}$ and $97.6 \pm 5.52 \Omega$, respectively, and the overall values of mean energy and lead impedance in the chest closed group were $1.67 \pm 1.00 \mathrm{~J}$ and $75.9 \pm 13.3 \Omega$, respectively. No complications were observed after wire removal. The gel pads became degraded and decreased in thickness, and signs of mild inflammation were evident on the gel pad. However, the gel pads did not elicit significant severe inflammatory reactions according to both gross and histologic assessments at 1 month after the surgery.

Conclusion: Atrial cardioversion using novel biodegradable gel pads that are easily affixed may afford a straightforward and effective treatment for atrial fibrillation after cardiac surgery.

A trial fibrillation (AF) is one of the most common complications after cardiac surgery. Postoperative incidence varies between $20 \%$ and $60 \%$ within the first week. ${ }^{1-5}$ The onset of postoperative AF might result in an increased risk for stroke, hemodynamic deterioration, prolonged hospital stay, and associated cost. ${ }^{6-8}$ 


\section{Abbreviations and Acronyms \\ $\mathrm{AF}=$ atrial fibrillation \\ $\mathrm{EPL}=$ epsilon polylysine \\ $\mathrm{HA}=$ hyaluronic acid \\ $\mathrm{POD}=$ postoperative day \\ $\mathrm{PV}=$ pulmonary vein}

Current treatment for postoperative AF consists of antiarrhythmic and anticoagulation drugs that may have considerable side effects. ${ }^{9}$ When AF results in hemodynamic instability that requires transthoracic cardioversion under general anesthesia, new arrhythmias, burns and pain to the patient, and problems associated with anesthesia might arise. Therefore, several investigators have attempted internal cardioversion using temporary epicardial defibrillation leads as an alternative to external transthoracic cardioversion to treat postoperative AF. ${ }^{10-14}$ The benefits of this therapy are a low-energy requirement for cardioversion, reduced myocardial damage from shock, minimized pain associated with energy delivery, and elective general anesthesia. However, securing leads on the right and left atria using S-, O-, or linear-shaped sutures is awkward and time-consuming, especially during off-pump surgery. Furthermore, extracting the leads increases the potential for bleeding.

Therefore, we developed a novel biodegradable biopolymer gel pad that directly attaches to the myocardial surface by electrostatic interaction. The custom-made cardioversion electrode consisted of a biodegradable gel pad and a spiral stainless steel wire (temporary myocardial lead, TME61Z, Dr Osypka, GMBH Medizintechnik, Rheinfelden-Herten, Germany) with an insulated portion (Figure 1). The present study examines the feasibility and effectiveness of low-energy internal cardioversion using our novel biodegradable gel pads.

\section{Materials and Methods Materials}

Hyaluronic acid sodium salt (HA: sodium salt; average molecular weight, $1150 \mathrm{kDa}$; produced by Streptococcus mutants of lactic acid bacteria) and poly ( $\epsilon$-L-lysine; EPL: average molecular weight, $3.8 \mathrm{kDa}$ ) were supplied by Chisso Co Ltd (Tokyo, Japan). We used 1-ethyl-3-(3-dimethylaminopropyl) carbodiimide hydrochloride (Dojindo Laboratories, Kumamoto, Japan), a water-soluble carbodiimide, as a crosslinker.

\section{Manufacture of Electrode Gel Pads}

Mixtures of $2 \mathrm{wt} \%$ each of HA and EPL (molar ratio of HA:EPL, 1:2) or $4 \mathrm{wt} \%$ each of HA and EPL (molar ratio of HA:EPL, 1:2) in double-distilled water were adjusted with $1 \mathrm{~N} \mathrm{HCl}$ to $\mathrm{pH}$ 7.0. A cardioversion wire with a spiral distal end was immersed in a circular Teflon (diameter, $40 \mathrm{~mm}$; depth, $4 \mathrm{~mm}$ ) mold containing the polyelectrolyte solutions, frozen in liquid nitrogen, and then freeze-dried using a lyophilizer (FreeZone 6, Labconco Corp, Kansas City, Mo). The cardioversion wire embedded in white solids was crosslinked by immersion in a mixture of $80 \mathrm{vol} \%$ ethanol and $20 \mathrm{vol} \%$ water containing $50 \mathrm{mmol} / \mathrm{L}$ water-soluble carbodiimide for 24 hours at $25^{\circ} \mathrm{C}$ and then washed in doubledistilled water. The chemical crosslinking is based on the activation of carboxylic sites with primary amine groups. The final product was a spongy, discoid HA-EPL crosslinked electrode gel pad $40 \mathrm{~mm}$ in diameter and $4 \mathrm{~mm}$ thick.

\section{Experimental Protocol}

The 12 pigs (weight, 30 to $42 \mathrm{~kg}$; average, $36.0 \pm 2.2 \mathrm{~kg}$ ) used in this study received humane care in accordance with the Principles of Laboratory Animal Care formulated by the National Society for Medical Research and the Guide for the Care and Use of Laboratory Animals prepared by the Institute of Laboratory Animal Resources, National Research Council (National Academy Press; revised 1996). AF ( $\geq 5$ minutes) was induced by pulmonary vein (PV) burst pacing and sustained in all 12 pigs. Phase I of this study investigated the feasibility of atrial defibrillation using the novel epicardial cardioversion gel pads with the chest open. Phase II examined the effectiveness of the novel epicardial cardioversion electrode gel pads implanted in a manner resembling clinical application.

\section{Operative Procedure}

The pigs were premedicated with an intramuscular injection of ketamine (10 $\mathrm{mg} / \mathrm{kg}$ body weight), intubated, and anesthetized with $0.5 \%$ to $2.5 \%$ halothane and a mixture of oxygen and nitrous oxide. Electrocardiography was continuously monitored using a polygraph system (RM-6000, Nihon Koden, Tokyo, Japan) throughout the procedure. The heart was exposed through a median sternotomy under general anesthesia, and 2 monopolar stainless steel temporary wire electrodes (temporary pacing wire, Johnson \& Johnson, NJ) were placed on the left PVs for pacing and recording. Two crosslinking biodegradable cardioversion electrode gel pads ( 2 and 4 wt $\%$ HAEPL) were respectively placed on the right appendage and left atria without suture (Figure 2). All wires were extruded through the skin and secured with a nonabsorbable suture.

\section{Atrial Defibrillation Protocols}

Phase I. We evaluated the effectiveness and safety of atrial defibrillation generated using the novel epicardial cardioversion electrode gel pads on the day of surgery with the chest open in 6 pigs ( $\mathrm{CO}$ group). With the use of a heating pad, the body temperature of the pigs was maintained within the normal physiologic range throughout the study. Electrocardiographic leads I, II, and III and electrograms from the left PV were monitored and recorded on a polygraph system. Sustained AF, defined as AF lasting for at least 5 minutes, was continuously induced by burst pacing at cycle lengths of $20 \mathrm{~ms}$ through the left PV electrodes using a customized fibrillator (Nihon Koden, Tokyo, Japan). We used an external programmer (Ventak ECD, model 2815, Guidant Corporation, Santa Clara, Calif) to control defibrillation that was delivered as an R-wave-synchronized biphasic shock to the atria through the cardioversion electrode gel pads. The shock intensity started at 0.5 $\mathrm{J}$ and was increased in 0.5- $\mathrm{J}$ increments to $3 \mathrm{~J}$ and thereafter in 1-J increments until AF was converted. This protocol was repeated 5 times per pig. The defibrillation threshold was defined as the lowest shock energy (voltage) required to convert AF into sinus rhythm. 


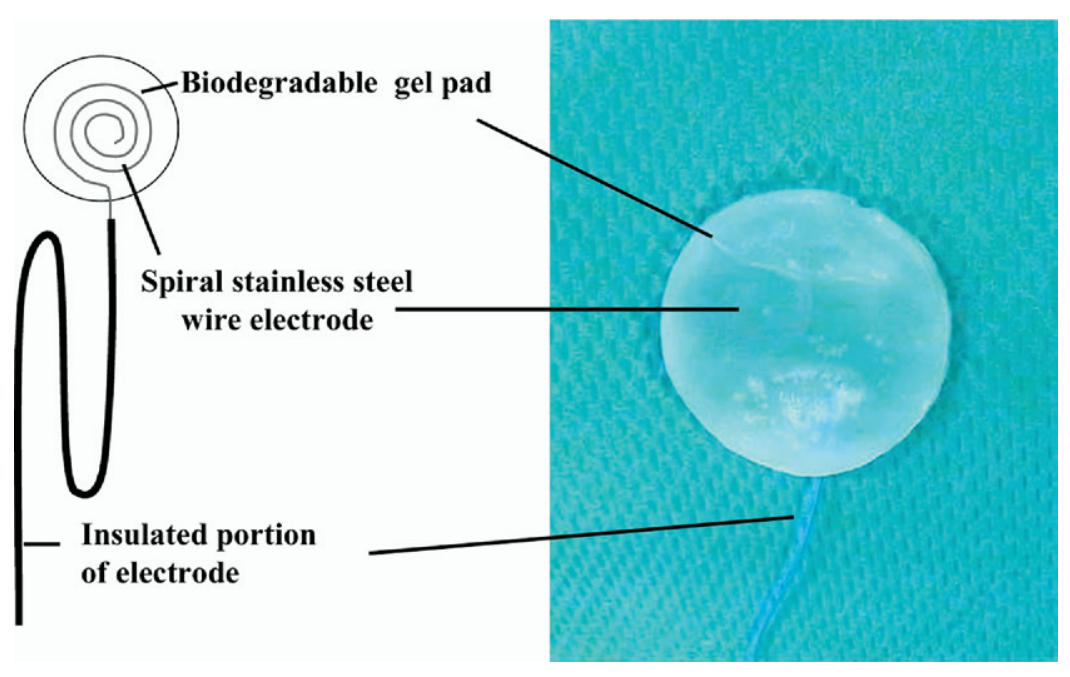

Figure 1. Biodegradable gel pads.

Phase II. A different group of 6 pigs underwent median sternotomy. The epicardial cardioversion electrode gel pad and pacing temporary wire electrodes were placed as described above for the first phase. Burst pacing was continuously applied from the PVs at cycle lengths of $20 \mathrm{~ms}$ to induce sustained AF. Cardioversion proceeded using a biphasic defibrillation system with the chest open. Shock intensity started at $0.5 \mathrm{~J}$ and was increased by $0.5 \mathrm{~J}$ to $1 \mathrm{~J}$, and thereafter $1-\mathrm{J}$ step increments were applied until 2 consecutive cardioversions were achieved at a single energy level. The defibrillation threshold was defined as the lowest shock energy (voltage) required to produce 2 consecutive cardioversions. Thereafter, the pericardium was reapproximated, the sternotomy was closed in the standard manner, and the pigs were allowed to recover. At postoperative days (PODs) 1, 3, 5, and 7, sustained AF was induced by burst pacing in the sedated state, and the defibrillation threshold was achieved with the chest closed as before. On POD 10, the cardioversion wires were removed from all pigs under sedation, but the electrode gel pads remained positioned around the heart. After predetermined time intervals, the heart was reexposed and the extent of degradation in vivo was histologically evaluated after sacrifice. Gel pads were removed from the pigs, fixed together with surrounding tissue in $10 \%$ formalin, sectioned using a microtome, and stained with hematoxylin-eosin.

\section{Statistical Analysis}

Data are reported as mean \pm standard deviation. The change over time of energy, impedance, and voltage were compared by Friedman's chi-square $r$ test followed by the Wilcoxon $t$ test with Bonferroni correction.

\section{Results}

\section{Phase I}

The gel pads were easily attached without suture in all 12 pigs. The epicardial cardioversion electrode gel pads positioned in 6 pigs did not migrate during this part of the study.

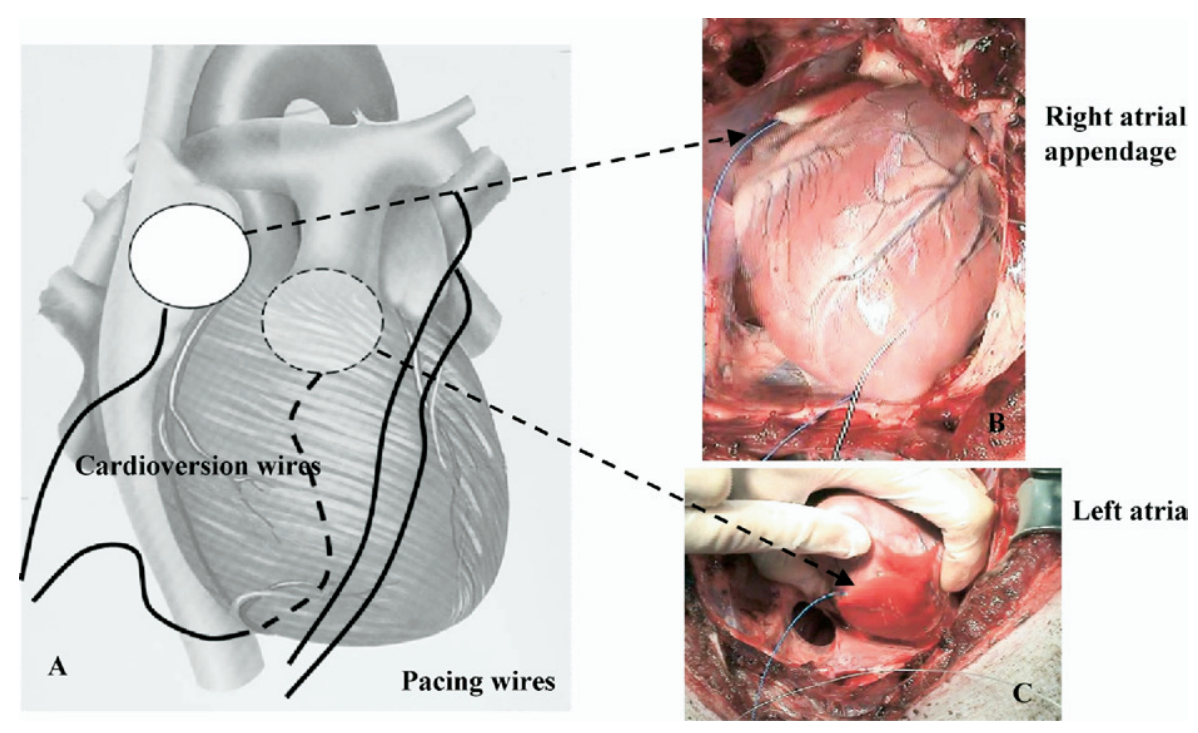

Figure 2. Location of 2 biodegradable gel pads for $A F$ in anterior $(A, B)$ and inferior (C) views of heart. 
TABLE 1. Mean values obtained at lowest defibrillation threshold for atrial fibrillation

\begin{tabular}{lccr}
\hline Animal No. & Energy (joules) & Impedance (ohms) & Voltage (volts) \\
\hline 1 & $0.60 \pm 0.20$ & $94.8 \pm 9.24$ & $95.0 \pm 15.0$ \\
2 & $0.70 \pm 0.24$ & $99.6 \pm 1.50$ & $104.6 \pm 16.0$ \\
3 & $0.60 \pm 0.20$ & $98.4 \pm 5.34$ & $94.8 \pm 15.1$ \\
4 & $0.70 \pm 0.24$ & $97.2 \pm 2.40$ & $102.4 \pm 18.1$ \\
5 & $0.70 \pm 0.24$ & $99.6 \pm 2.33$ & $102.0 \pm 17.6$ \\
6 & $0.60 \pm 0.20$ & $96.2 \pm 5.78$ & $94.8 \pm 14.6$ \\
Total & $0.65 \pm 0.23$ & $97.6 \pm 5.52$ & $98.9 \pm 16.6$
\end{tabular}

Means \pm SD

The induced AF was terminated in all 6 pigs while the chest was open by delivering an atrial shock as follows: mean energy, $0.65 \pm 0.23 \mathrm{~J}$; mean impedance, $97.6 \pm 5.52 \Omega$; mean voltage, $98.9 \pm 16.6 \mathrm{~V}$ (Table 1). Figure 3 shows a representative example.

\section{Phase II}

Table 2 describes the mean values obtained at the defibrillation threshold over the postoperative course of 1 week in another 6 pigs. The defibrillation threshold was achieved with the chest open at POD 0 and with the chest closed at PODs 1, 3, 5, and 7. Table 2 shows the mean energy and lead impedance obtained at the defibrillation threshold. Energy and voltage increased over time. Impedance significantly differed between POD 0 and the other days, perhaps because of the chest being open or closed. All induced AF was cardioverted to sinus rhythm over a period of 1 week after surgery. No early recurrence of AF was evident within 1 minute after a primary cardioversion. On POD 10, all cardioversion wires were easily removed by the simple transcutaneous traction that is used to remove temporary cardiac pacing wires. No complications were related to lead extraction, and no bleeding was detected when the chest was inspected after the animal was sacrificed at the predetermined POD.

Gross and histologic assessment at 1 month after the surgery confirmed that the gel pads dissolved. Mild inflam- mation predominantly comprised lymphocyte with foreign body giant cells on the gel pad. Phagocytes of the gel fragments absorbed by foreign body giant cells were often identified. Mild fibrosis with fibroblast infiltration was also evident on the gel pad. Inflammation was milder in the epicardium beneath than on the gel pad. In the myocardium beneath the gel pad, few inflammatory cells were evident and the degree of inflammatory reactions was similar to that of other epicardium and myocardium of the same heart that did not contact the gel pad (Figure 4).

\section{Discussion}

Postoperative AF is often regarded as a benign clinical condition, but it has been associated with adverse outcomes, including patient discomfort and anxiety, increased risk for stroke, hemodynamic instability, prolonged hospital stay, and increased hospital costs. ${ }^{6-8}$ A reduction in postoperative AF was recently identified in an off-pump series, although this remains an area of controversy. ${ }^{2}$ Nonetheless, off-pump coronary artery bypass grafting surgery has not eliminated the problem of postoperative AF. Currently applied antiarrhythmics and anticoagulation might induce considerable side effects, such as new arrhythmias. If all patients receive routine prophylaxis, then approximately $70 \%$ of them would be exposed to unnecessary treatment and possible undesirable side effects. Transthoracic cardioversion also has the side effect of causing new arrhythmias, burns, and pain, as well as problems with anesthesia. Despite improvements in technical advances, myocardial protection, cardiopulmonary bypass, and treatment over the last 50 years in cardiac surgery, the incidence of postoperative AF has remained unchanged.

This study demonstrated that atrial defibrillation using temporary epicardial cardioversion gel pads placed on the right and left atria in a pig model can be effective and safe, and use remarkably little energy not only with the chest open intraoperatively (phase I) but also with the chest closed postoperatively (phase II) as an alternative therapy to postoperative AF. In addition, we evaluated a physiologic pericarditis model of AF that was suggested

\section{Atrial fibrillation Shock Sinus rhythm}

ECG I

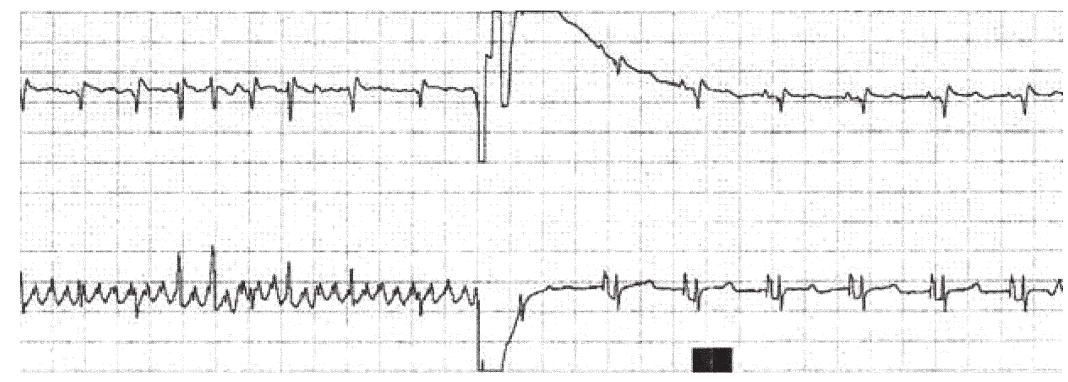

Figure 3. Example of atrial defibrillation in pig model of pericarditis with biodegradable cardioversion gel pads. $E C G$, electrocardiogram; PV, pulmonary vein. 
TABLE 2. Changes over time of mean values obtained at defibrillation threshold for atrial fibrillation

\begin{tabular}{lcccccc}
\hline & POD & $\mathbf{0}$ & $\mathbf{1}$ & $\mathbf{5}$ & $\mathbf{7}$ & Overall values \\
\hline Energy $(\mathrm{J})$ & $0.83 \pm 0.24$ & $1.00 \pm 0.50$ & $1.83 \pm 0.90$ & $2.00 \pm 1.00$ & $2.67 \pm 0.94$ & $1.67 \pm 1.00$ \\
Impedance $(\Omega)$ & $100.3 \pm 6.69$ & $67.4 \pm 5.19^{*}$ & $69.4 \pm 5.79^{*}$ & $68.9 \pm 3.75^{*}$ & $73.7 \pm 3.66^{*} \dagger$ & $75.9 \pm 13.3$ \\
Voltage $(\mathrm{V})$ & $111.6 \pm 17.8$ & $116.9 \pm 28.2$ & $157.1 \pm 37.7$ & $164.3 \pm 39.8^{*}$ & $192.4 \pm 37.3^{*}+\ddagger$ & $148.5 \pm 45.0$ \\
\hline
\end{tabular}

POD, Postoperative day. ${ }^{*}$ Versus POD 0 value $(<.05)$. †Versus POD 1 value $(<.05)$. \pm Versus POD 3 value $(<.05)$. Means \pm SD.

by the common occurrence of atrial arrhythmias in patients after cardiac surgery and their association with the time course of pericarditis. ${ }^{15,16}$ We also defined the defibrillation threshold as the lowest shock required to elicit 2 consecutive cardioversions, because no single amount of energy can be defined as an absolute threshold and the upper boundary of the defibrillation curve becomes more accurately defined, allowing smaller efficacy margins to ensure $100 \%$ defibrillation. ${ }^{17}$ This clinical counterpart renders the model reasonably relevant to patients after cardiac surgery in terms of both the arrhythmia mechanism and the time course, and thus is a logical choice of therapy for postoperative AF. ${ }^{18}$

Our gel pad consists of cationic poly ( $\epsilon$-lysine) and anionic HA, which is an important component of the extracellular matrix and connective tissues such as cartilage. HA has been applied for medical purposes as a viscoelastic biomaterial in ophthalmologic surgery and as a viscosupplementation agent for the treatment of joint disease in orthopedics. ${ }^{19-22}$ In addition, HA is included in cosmetics because of its high water-retention capacity and in drug-delivery systems because of its biodegradability. The biocompatible counter polycation of poly ( $\epsilon$-lysine) can be easily conjugated with bioactive mole- cules. ${ }^{23}$ Furthermore, EPL is widely applied to promote cell adhesion to solid substrates. Schwann cell attachment significantly increases when crosslinked HA strands are coated with polylysine. ${ }^{24} \mathrm{We}$ applied the cell adhesion properties of polyelectrolyte gels with HA and EPL to tissue surface attachment and constructed a biocompatible, biodegradable gel pad.

\section{Clinical Implications}

This pilot study demonstrates the potential clinical utility of low-energy cardioversion using biodegradable electrode gel pads in the management of AF in patients after cardiac surgery. Instead of external cardioversion requiring a general anesthetic, which often causes cardiac depression in patients with already diminished cardiac output and coronary blood flow, only mild sedation is required, and internal cardioversion can be used to restore sinus rhythm. Lowenergy internal cardioversion might be an alternative in patients with high transthoracic impedance, such as those who are obese or have chronic obstructive lung disease. This therapy would provide a quick method of termination of AF without having to resort to a large external cardioversion shock or antiarrhythmic drugs.

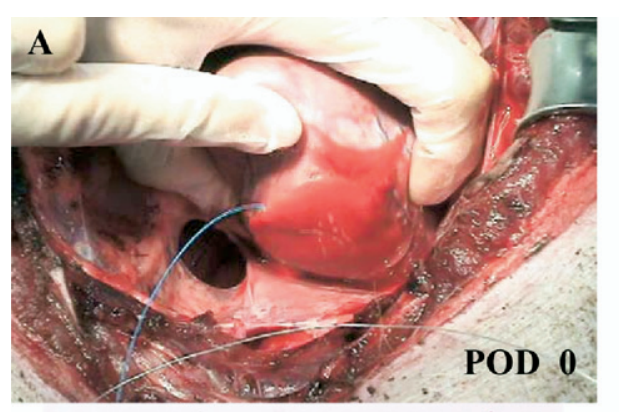

C

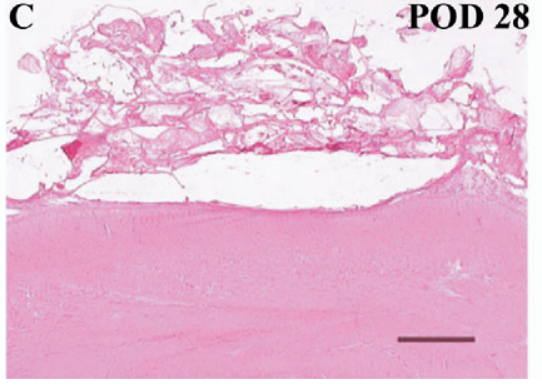

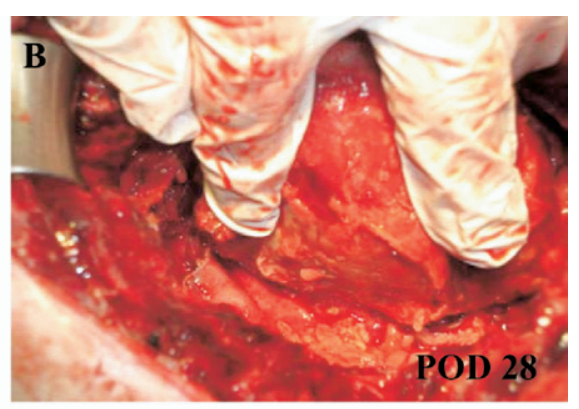

D

POD 28

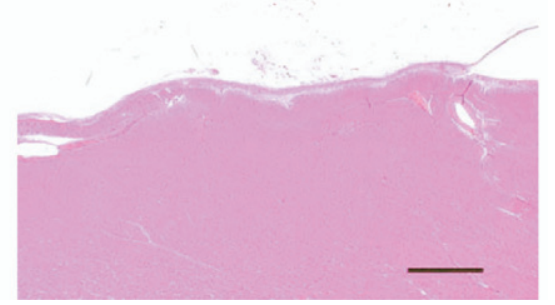

Figure 4. Gross (A, B) and histologic (C, D) assessments 1 month after surgery. Bar $=1000 \mu \mathrm{m}$. Epicardium and myocardium beneath (C) and without (D) gel pads. POD, Postoperative day. 
Recent pilot studies using temporary epicardial wire electrodes placed on the right and left atria of patients demonstrated that atrial defibrillation can be accomplished by shocks at low energy ranging from 5 to $6 \mathrm{~J}^{10,12,13}$ Low-energy cardioversion using biodegradable gel pads can be performed without complicated and time-consuming lead placement, which is required in cardioversion using temporary epicardial wire electrodes, and without risk of bleeding after removing the wire because of its biodegradability. Although low-energy shocks with biodegradable gel pads cardioverted the atria in our animal model, it remains unknown whether the shock energies as low as $2 \mathrm{~J}$ that worked in pigs will be as successful in human beings. We did not evaluate the correlation between energy level and pain threshold using this system. Other systems using temporary epicardial defibrillation wire electrodes have defibrillated the atria with low-energy shocks. When energy shocks as low as $1 \mathbf{~ J}$ are delivered, shock-related discomfort is experienced by most patients. Therefore, whether this system can be tolerated without sedation remains uncertain. Although general anesthesia might be elective, mild sedation might be required to reduce patient discomfort. However, a significant portion of patients are at risk for the development of AF after cardiac surgery. Such patients might benefit from the use of biodegradable gel pads that are easy to affix, especially during off-pump surgery, if they can provide effective and safe low-energy atrial cardioversion. We suggest that the utility of these pads be examined in patients.

\section{Study Limitations}

The main limitation of this study is that AF was induced in normal pig hearts. The substrates might differ between humans and pigs. In addition, the pigs had not undergone a bypass and did not have atriotomy. Therefore, it remains to be seen whether diseased hearts with AF in the clinical setting will respond like the pig hearts. However, studies of animals and patients with epicardial defibrillation wire electrodes placed on the right and left atria showed that AF can be converted to sinus rhythm with low-energy shocks. ${ }^{10-14}$ The results of these pilot studies with epicardial defibrillation wire electrodes have been encouraging and indicate that similar success using lower-energy shocks is achievable in patients using the biodegradable gel pads. Nonetheless, because little is known about the toxicity of the biocompatible gel pad, further animal studies in vivo are required.

\section{Conclusions}

The amount of energy required for atrial defibrillation using novel biodegradable cardioversion gel pads in a pig model was well less than $2 \mathrm{~J}$. The advantages of this system are easy placement, straightforward removal of the wires, and quick and safe atrial shock application. Atrial cardioversion using biodegradable gel pads that are easy to affix may comprise a straightforward and effective treatment for $\mathrm{AF}$ after cardiac surgery.

We thank Drs Isao Matsumoto and Hideki Takeda for their helpful suggestions and valuable technical assistance in conducting the study and Drs Misato Kasashima and Yoko Tanaka for their helpful suggestions and assistance in examining the histologic specimens.

\section{References}

1. Zaman AG, Archbold RA, Helft G, Paul EA, Curzen NP, Mills PG. Atrial fibrillation after coronary artery bypass surgery: a model for preoperative risk stratification. Circulation. 2000;101:1403-8.

2. Hakala T, Berg E, Hartikainen JE, Hippelainen MJ. Intraoperative high-rate atrial pacing test as a predictor of atrial fibrillation after coronary artery bypass surgery. Ann Thorac Surg. 2002;74:2072-5.

3. Yazicioglu L, Eryilmaz S, Sirlak M, Inan MB, Aral A, Tasoz R, et al. The effect of preoperative digitalis and atenolol combination on postoperative atrial fibrillation incidence. Eur J Cardiothorac Surg. 2002; 22:397-401.

4. Forlani S, De Paulis R, de Notaris S, Nardi P, Tomai F, Proietti I, et al. Combination of sotalol and magnesium prevents atrial fibrillation after coronary artery bypass grafting. Ann Thorac Surg. 2002;74: $720-6$.

5. Aranki SF, Shaw DP, Adams DH, Rizzo RJ, Couper GS, VanderVliet $\mathrm{M}$, et al. Predictors of atrial fibrillation after coronary artery surgery. Current trends and impact on hospital resources. Circulation. 1996; 94:390-7.

6. Creswell LL, Schuessler RB, Rosenbloom M, Cox JL. Hazards of postoperative atrial arrhythmias. Ann Thorac Surg. 1993;56:539-49.

7. Almassi GH, Schowalter T, Nicolosi AC, Aggarwal A, Moritz TE, Henderson WG, et al. Atrial fibrillation after cardiac surgery: a major morbid event? Ann Surg. 1997;226:501-13.

8. Kowey PR, Dalessandro DA, Herbertson R, Briggs B, Wertan MA, Rials SJ, et al. Effectiveness of digitalis with or without acebutolol in preventing atrial arrhythmias after coronary artery surgery. Am J Cardiol. 1997;79:1114-7.

9. Creswell LL, Damiano RJ Jr. Postoperative atrial fibrillation: an old problem crying for new solutions. J Thorac Cardiovasc Surg. 2001; 121:638-41.

10. Patel AN, Hamman BL, Patel AN, Hebeler RF, Wood RE, Cockerham CA, et al. Epicardial atrial defibrillation: successful treatment of postoperative atrial fibrillation. Ann Thorac Surg. 2004;77:831-7.

11. Cmolik BL, Oritz J, Ayers GM, Lee JH, Geha AS, Waldo AL. Successful atrial defibrillation with very-low-energy shocks by means of temporary epicardial wire electrodes. J Thorac Cardiovasc Surg. 1996;111:392-8.

12. Kleine P, Blommaert D, van Nooten G, Blin O, Haisch G, Stoffelen W, et al. Multicenter results of TADpole heart wire system used to treat postoperative atrial fibrillation. Eur J Cardiothorac Surg. 1999;15: 525-7.

13. Liebold A, Wahba A, Birnbaum DE. Low-energy cardioversion with epicardial wire electrodes: new treatment of atrial fibrillation after open heart surgery. Circulation. 1998;98:883-6.

14. Ortiz J, Sokoloski MC, Ayers GM, Cmolik BL, Niwano S, Geha AS, et al. Atrial defibrillation using temporary epicardial defibrillation stainless steel wire electrodes: studies in the canine sterile pericarditis model. J Am Coll Cardiol. 1995;26:1356-64.

15. Waldo AL, MacLean WAH. Diagnosis and Treatment of Cardiac Arrhythmias Following Open Heart Surgery. Mount Kisco, NY: Futura; 1980:115-29.

16. Page PL, Plumb VJ, Okumura K, Waldo AL. A new animal model of atrial flutter. J Am Coll Cardiol. 1986;8:872-9. 
17. Singer I, Lang D. Defibrillation threshold: clinical utility and therapeutic implications. Pacing Clin Electrophysiol. 1992;15:932-49.

18. Ortiz J, Igarashi M, Gonzalez HX, Johnson NJ, Waldo AL. A new, reliable atrial fibrillation model with a clinical counterpart. J Am Coll Cardiol. 1993;21:183A.

19. Balazs EA, Denlinger JL. Viscosupplementation: a new concept in the treatment of osteoarthritis. J Rheumatol Suppl. 1993;39:3-9.

20. Balazs EA. In: Miller D, Stegmann R, eds. Healon (Sodium Hyaluronate). A Guide to its Use in Ophthalmic Surgery. New York: Wiley; 1983:5-28.

21. Kim SJ, Hahn SK, Kim MJ, Kim DH, Lee YP. Development of a novel sustained release formulation of recombinant human growth hormone using sodium hyaluronate microparticles. $J$ Control Release. 2005;104:323-35. Epub 2005 Apr 7.

22. Ohri R, Hahn SK, Hoffman AS, Stayton PS, Giachelli CM. Hyaluronic acid grafting mitigates calcification of glutaraldehyde-fixed bovine pericardium. J Biomed Mater Res A. 2004;70:328-34.

23. Picart C, Lavalle P, Hubert P, Cuisinier FJG, Decher F, Schaaf P, et al. Buildup mechanism for poly(L-lysine)/hyaluronic acid films onto a solid surface. Langmuir. 2001;17:7414-24.

24. Hu M, Sabelman EE, Tsai C, Tan J, Hentz VR. Improvement of Schwann cell attachment and proliferation on modified hyaluronic acid strands by polylysine. Tissue Eng. 2000;6:585-93.

Access to The Journal of Thoracic and Cardiovascular Surgery Online is reserved for print subscribers!

Full-text access to The Journal of Thoracic and Cardiovascular Surgery Online is available for all print subscribers. To activate your individual online subscription, please visit The Journal of Thoracic and Cardiovascular Surgery Online, point your browser to http://www.mosby.com/jtcvs, follow the prompts to activate your online access, and follow the instructions. To activate your account, you will need your subscriber account number, which you can find on your mailing label (note: the number of digits in your subscriber account number varies from 6 to 10 ). See the example below in which the subscriber account number has been circled:

\section{Sample mailing label}

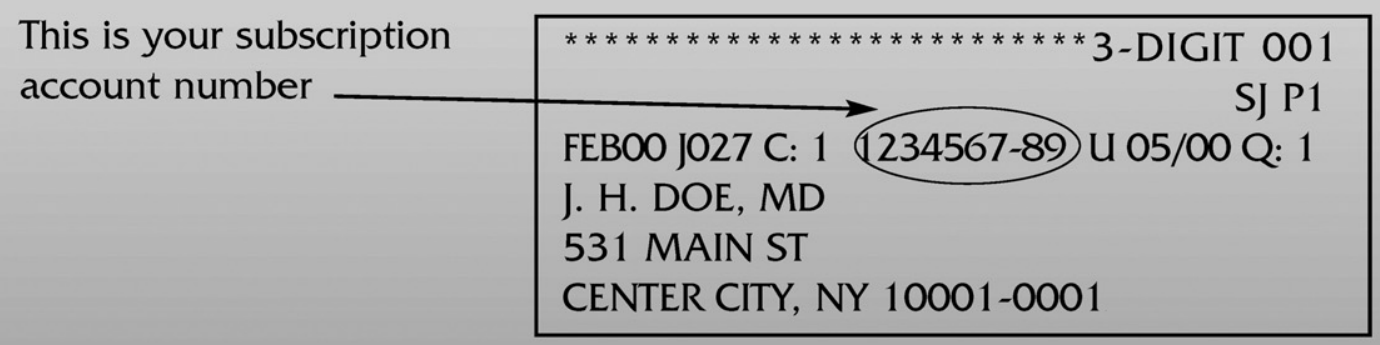

Personal subscriptions to The Journal of Thoracic and Cardiovascular Surgery Online are for individual use only and may not be transferred. Use of The Journal of Thoracic and Cardiovascular Surgery Online is subject to agreement to the terms and conditions as indicated online. 